\title{
AMINO ACIDS METABOLISM AND DEgRADATION IS REGULATED DURING PORCINE OVIDUCTAL EPITHELIAL CELLS (OECS) PRIMARY CULTURE IN VITRO - A SIGNALING PATHWAYS ACTIVATION APPROACH
}

Wiesława Kranc ${ }^{1}$, Maurycy Jankowski ${ }^{1}$, Joanna Budna ${ }^{2}$, Piotr Celichowski ${ }^{2}$, Ronza Khozmi ${ }^{1}$, Artur Bryja $^{1}$, Sylwia Borys ${ }^{1}$, Marta Dyszkiewicz-Konwińska ${ }^{1,3}$, Michal Jeseta ${ }^{4}$, Magdalena Magas ${ }^{1,5}$, Dorota Bukowska $^{5}$, Paweł Antosik ${ }^{5}$, Klaus P. Brüssow ${ }^{5}$, Małgorzata Bruska ${ }^{1}$, Michał Nowicki ${ }^{2}$, Maciej Zabel $^{2,6}$, Bartosz Kempisty ${ }^{1,2,4 *}$

\begin{abstract}
The ovary is part of the reproductive system, possessing very important functions in the reproduction process (ovum and embryo transfer, providing a suitable environment for sperm capacitation, etc.). There are two types of cells in the fallopian tubes: alveolar and secretive cells. These study shows the metabolic processes in pig oviductal epithelial cells associated with the activation of signaling pathways of amino acids metabolism and degradation during long-term in vitro culture. Oviductal epithelial cells from 45 colonies in the anestrous phase of the estrous cycle have been utilized in this study. RNA extract from the OEC primary cultures was pooled after $24 \mathrm{~h}, 7$ days, 15 days and 30 days from the beginning of culture and the transcriptome investigated by Affymetrix ${ }^{\circledR}$ Porcine Gene 1.1 ST. From the whole transcript that consisted of 2009 different genes, 1537 were upregulated and 995 were downregulated after 7 days of culture, 1471 were upregulated and 1061 were downregulated after 15 days of culture and 1329 were upregulated and 1203 were downregulated after 30 days of culture. The results of these studies provide, for the first time, information on the activation of metabolic pathways of amino acids such as valine, leucine, isoleucine, cysteine, and methionine in the investigated tissue. They also indicate genes that may be OECs-specific genetic markers that are expressed or upregulated during long-term in vitro culture.
\end{abstract}

Running title: regulation of amino acid signaling pathways

Keywords: pig, oviductal epithelial cells, in vitro culture (IVC), signaling pathways

\footnotetext{
${ }^{1}$ Department of Anatomy, Poznan University of Medical Sciences, Poznan, Poland

${ }^{2}$ Department of Histology and Embryology, Poznan University of Medical Sciences, Poznan, Poland

${ }^{3}$ Department of Biomaterials and Experimental Dentistry, Poznan University of Medical Sciences, Poznan, Poland

${ }^{4}$ Department of Obstetrics and Gynecology, University Hospital and Masaryk University, Obilni trh 11, 60200 Brno, Czech Republic

${ }^{5}$ Veterinary Center, Nicolaus Copernicus University in Toruń, Torun, Poland

${ }^{6}$ Department of Histology and Embryology, Wroclaw Medical University, Wroclaw, Poland

* Correspondence: bkempisty@ump.edu.pl

Full list of author information is available at the end of article 


\section{Introduction}

It was well found that domestic pigs are a useful model for many aspects of biomedical research. Indeed, phylogenetical similarities between pig and human open a new gateway in xenotransplantation, especially with regard to heart disease, orthopedics and traumatology. Although, transgenic pig technology is well established, there are still many difficulties before a successful xenotransplant can be performed. Therefore, stem cells technology was built as the basis of regenerative a deconstructive bio-medicine. Moreover, application of a stem cells was recently used as the main tool of advanced biotechnology [1]-[3].

Primary, in vitro cell cultures (IVC) are one of the most prospective methods of tissue and organ regeneration, with a large probability for application in reconstructive medicine. However, despite the fact that the stem-like specificity of cells cultured primarily in vitro is well recognized, the cellular physiology as well as molecular properties, are not yet properly determined [4]-[6]. Our recent experiments indicate the huge plasticity of porcine cells of origin associated with reproduction, such as ovarian granulosa cells, and/or endometrial cells, cultured primary in vitro for short,- or long-term [5], [7]. However, the differences in cellular proliferation and differentiation capability between various cell types are significant, depending substantially on the type of reproductive tissue and organ from which they are isolated. Furthermore, physiology of the cells, represented by genetically programed lifespan, ability for survival and/or apoptosis differs significantly, even in tissues recovered from the same donor. In result, each cell primary in vitro culture provides information about tissue dependent specificity and plasticity

We have also shown that porcine oviductal epithelial cells (OECs) differ significantly from endometrial cells and ovarian granulosa cells in the logarithmic phase of in vitro proliferation. It may be suggested that these cells cultured primary in vitro displayed different proliferation capability, which is in tissue type dependent manner. Although the in vitro primary culture system of OECs was well established by our team, the physiological and molecular properties of these cells are still not entirely known (unpublished data).

In this study, using Affymetrix microarray assays, we investigated the expression profile of genes involved in several important processes of amino acids metabolism. In results, we presented proteins - and enzymes significantly associated with activation of signaling pathways, involved in cysteine and methionine metabolism as well as valine, leucine and isoleucine degradation.

\section{Material and Methods Animals}

In this study, crossbred gilts $(n=45)$ at the age of about nine months and which displayed two regular estrous cycles were collected from a commercial herd.
All the animals were checked daily for estrus behavior and were slaughtered after reaching the anestrus phase of the estrus cycle. The uteri were then transported to the laboratory within $30 \mathrm{~min}$ at $38^{\circ} \mathrm{C}$.

\section{RNA extraction from oviductal epithelial cell}

Oviductal epithelial cell were pooled and harvested 24h, 7 days, 15 days and 30 days after beginning of culture. Total RNA was extracted from samples using TRI Reagent (Sigma, St Louis, MO, USA) and RNeasy MinElute cleanup Kit (Qiagen, Hilden, Germany). The amount of total mRNA was determined from the optical density at $260 \mathrm{~nm}$, and the RNA purity was estimated using the 260/280 nm absorption ratio (higher than 1.8) (NanoDrop spectrophotometer, Thermo Scientific, ALAB, Poland). The RNA integrity and quality were checked on a Bioanalyzer 2100 (Agilent Technologies, Inc., Santa Clara, CA, USA). The resulting RNA integrity numbers (RINs) were between 8.5 and 10 with an average of $9.2(\mathrm{Ag}$ ilent Technologies, Inc., Santa Clara, CA, USA). The RNA in each sample was diluted to a concentration of $100 \mathrm{ng} / \mu \mathrm{l}$ with an OD260/OD280 ratio of 1.8/2.0. From each RNA sample, 500 ng of RNA was taken for microarray expression assays.

\section{Microarray expression analysis and statistics}

Total RNA (100 ng) from each pooled sample was subjected to two rounds of sense cDNA amplification (Ambion ${ }^{\circledR}$ WT Expression Kit). The obtained cDNA was used for biotin labeling and fragmentation by Affymetrix GeneChip ${ }^{\circledR}$ WT Terminal Labeling and Hybridization (Affymetrix). Biotin-labeled fragments of cDNA $(5.5 \mu \mathrm{g})$ were hybridized to the Affymetrix ${ }^{\circledR}$ Porcine Gene 1.1 ST Array Strip $\left(48^{\circ} \mathrm{C} / 20 \mathrm{~h}\right)$. Microarrays were then washed and stained according to the technical protocol using the Affymetrix GeneAtlas Fluidics Station. The array strips were scanned employing Imaging Station of the GeneAtlas System. Preliminary analysis of the scanned chips was performed using Affymetrix GeneAtlasTM Operating Software. The quality of gene expression data was confirmed according to the quality control criteria provided by the software. The obtained CEL files were imported into downstream data analysis software.

All of the presented analyses and graphs were performed using Bioconductor and R programming languages. Each CEL file was merged with a description file. In order to correct background, normalize, and summarize results, we used the Robust Multiarray Averaging (RMA) algorithm. To determine the statistical significance of the analyzed genes, moderated $t$-statistics from the empirical Bayes method were performed. The obtained $\mathrm{p}$-value was corrected for multiple comparisons using Benjamini and Hochberg's false discovery rate. The selection of significantly altered genes was based on a p-value beneath 0.05 and expression higher than two fold. 
Differentially expressed genes were subjected selection by examination of genes involved in cell migration regulation. The differentially expressed gene list (separated for up- and down-regulated genes) was uploaded to DAVID software (Database for Annotation, Visualization and Integrated Discovery) [8], where genes belonging to the "valine, leucine and isoleucine degradation" and "cysteine and methionine metabolism" pathways from Kyoto Encyclopedia of Genes and Genomes (KEGG) were obtained. Subsequently the expression levels of that genes were marked on the pathways using a "pathview" - tool set for pathway based data integration and visualization [9]. Expression data of these genes were also subjected to a hierarchical clusterization procedure, and their expression values were presented as a heat map.

Interactions between differentially expressed genes/proteins belonging to the "valine, leucine and isoleucine degradation" and "cysteine and methionine metabolism" KEGG pathways were investigated by STRING10 software (Search Tool for the Retrieval of Interacting Genes) [10]. The list of gene names was used as a query for an interaction prediction. The search criteria were based on co-occurrences of genes/proteins in scientific texts (text mining), co-expression, and experimentally observed interactions. The results of such analyses

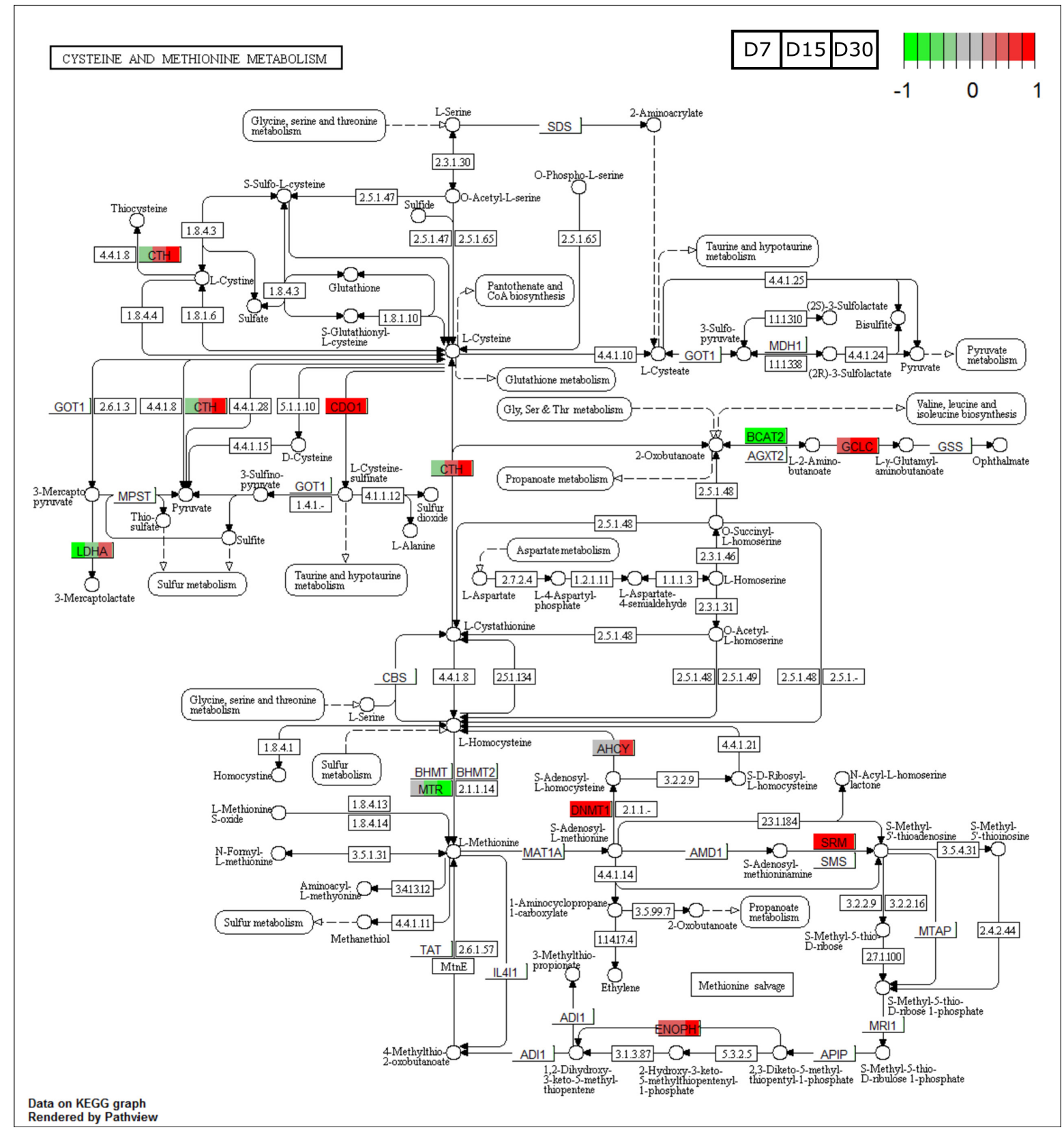

Figure 1 The "cysteine and methionine metabolism" KEGG pathway with marked expression levels of differently expressed genes. Arbitrary signal intensity acquired from microarray analysis is represented by colors (green, higher; red, lower expression). The boxes with names of genes were separated for three parts with representation of gene expression from the 7,15 and 30 day of culture 
generated a gene/protein interaction network where the intensity of the edges reflected the strength of the interaction score.

Ethical approval: The research related to animals use has been complied with all the relevant national regulations and institutional policies for the care and use of animals. Bioethical Committee approval no. 32/2012.

\section{Results}

To investigate Oviductal epithelial cell transcriptome changes following 7,15 and 30 days after start of primary porcine oviductal epithelial cells, we performed whole gene expression analysis by Affymetrix ${ }^{\circledR}$ Porcine Gene 1.1 ST Array. In such assay expression of more than 14789 porcine transcripts was examined. The genes for which the fold change was higher than the cut-off value (fold $>|2|$ ) and corrected $\mathrm{p}$ value $<0.05$, were considered as differentially expressed. From the whole transcript that consist of 2552 different genes. From these genes 1537 were upregulated and 995 were downregulated after 7 days of culture, 1471 were upregulated and 1061 were downregulated after 15 days of culture and 1329 were upregulated and 1203 were downregulated after 30 days of culture.

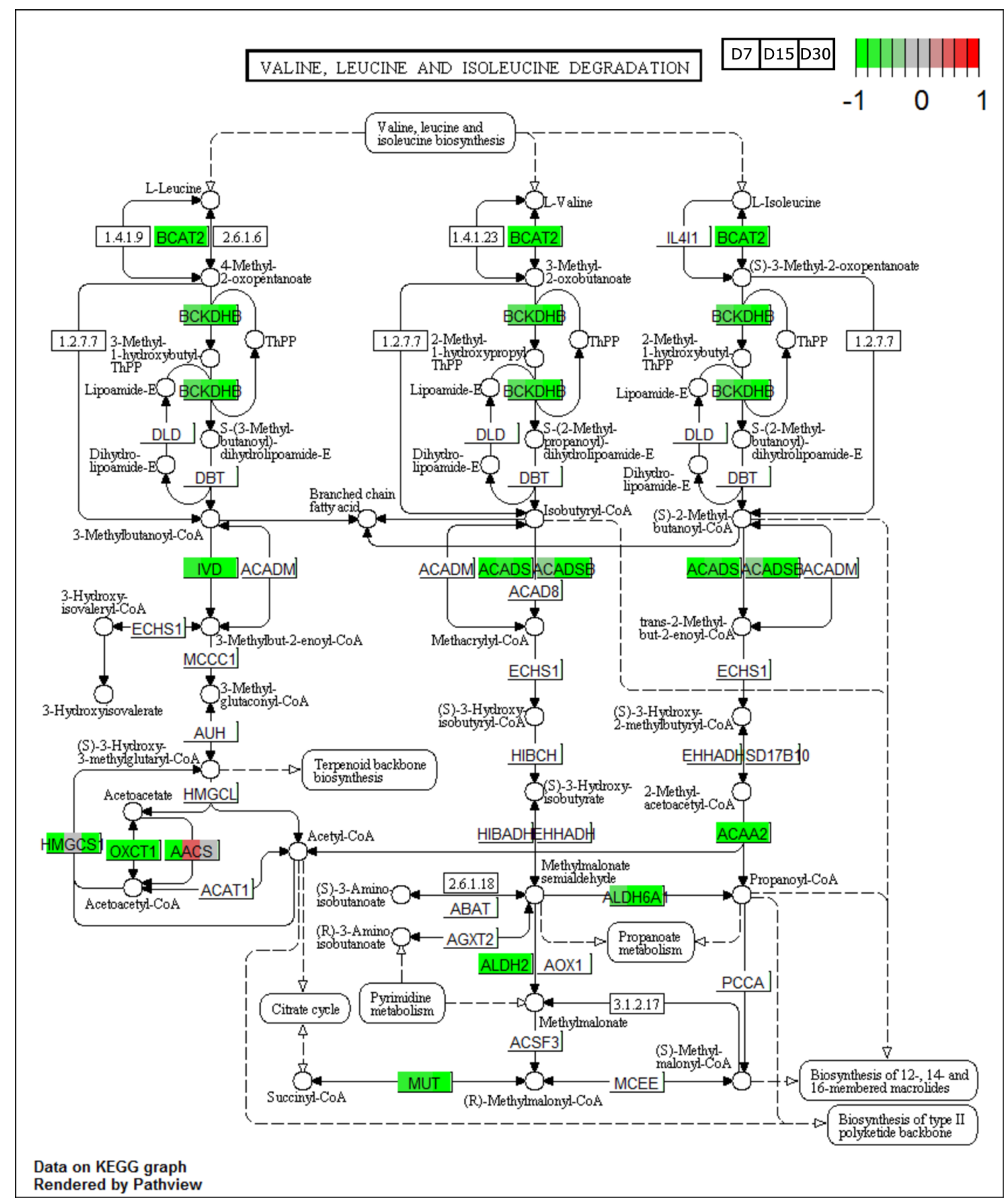

Figure 2 The "valine, leucine and isoleucine degradation" KEGG pathway with marked expression levels of differently expressed genes. Arbitrary signal intensity acquired from microarray analysis is represented by colors (green, higher; red, lower expression). The boxes with names of genes were separated for three parts with representation of gene expression from the 7,15 and 30 day of culture 
Among these genes, genes belong to the "valine, leucine and isoleucine degradation" and "cysteine and methionine metabolism" KEGG pathways were extracted by DAVID (Database for Annotation, Visualization and Integrated Discovery) software. Subsequently the logarithm from expression values of these genes were marked on these pathways using "pathview" tools set (Fig. 1 and Fig. 2). Up and down regulated gene sets were subjected to DAVID searching separately and only gene sets, where adj. $p$ value was lower than 0.05 were selected (Tab. 1). These two sets of genes was subjected to hierarchical clusterization procedure and presented as heatmap (Fig. 3).

In order to further explore known interaction between genes of interest, STRING-generated interaction network was generated among differentially

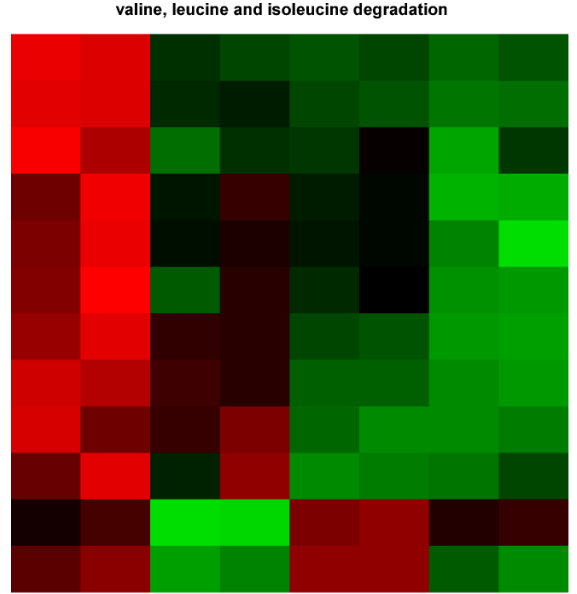

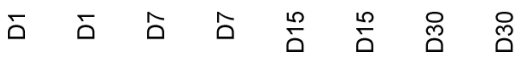

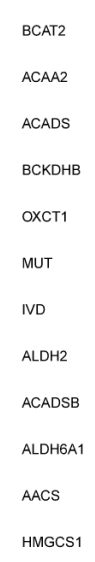

西

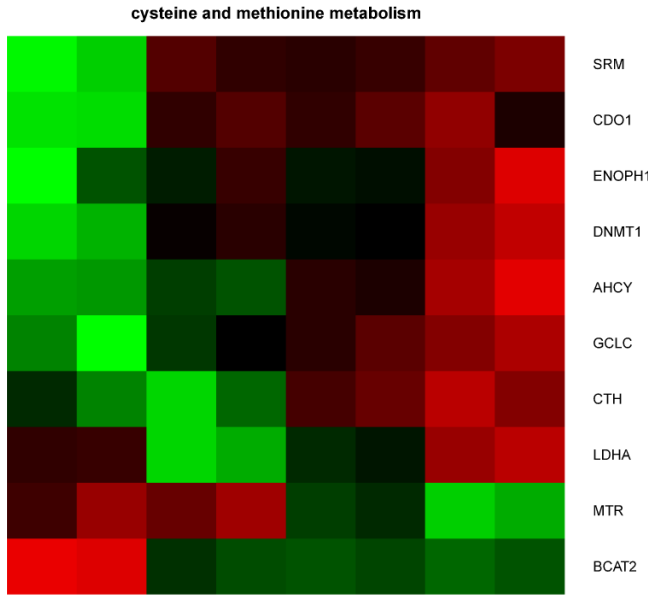

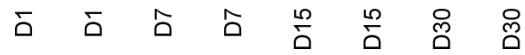

Figure 3 Heat map representation of differentially expressed genes belonging to the "cysteine and methionine metabolism" and "valine, leucine and isoleucine degradation" KEGG pathways. Arbitrary signal intensity acquired from microarray analysis is represented by colors (green, higher; red, lower expression). log2 signal intensity values were resized to Row Z-Score scale for any single genes (from -2, the lowest expression to +2 , the highest expression)

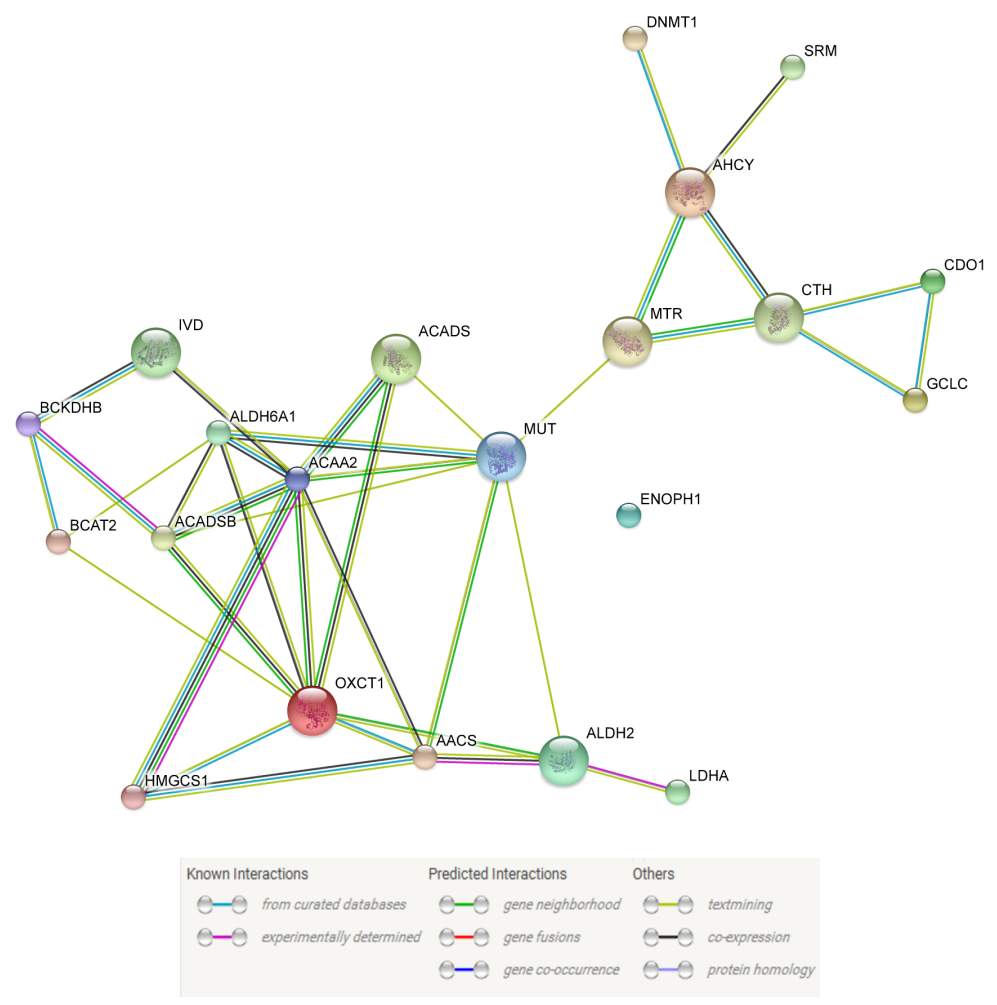

Figure 4 STRING-generated interaction network between differentially expressed genes, belonging to the "cysteine and methionine metabolism" and "valine, leucine and isoleucine degradation" KEGG pathways. Applied prediction methods: text mining, co-expression, experimentally observed interactions 
Table 1 Fold changes, adjusted p values and ENTREZ gene ID of differentially expressed genes belonging to the "cysteine and methionine metabolism" and "valine, leucine and isoleucine degradation" KEGG pathways. Symbols and names of the selected genes are also shown

valine, leucine and isoleucine degradation

\begin{tabular}{|c|c|c|c|c|c|c|c|}
\hline Offical gene symbol & fold ratio $7 \mathrm{D} / 24 \mathrm{~h}$ & fold ratio $15 \mathrm{D} / 24 \mathrm{~h}$ & fold ratio $30 \mathrm{D} / 24 \mathrm{~h}$ & adj.p.value $D 7 / 24 h$ & adj.p.value D15/24h & adj.p.value $\mathrm{D} 30 / 24 \mathrm{~h}$ & ENTREZ GENE ID \\
\hline HMGCS1 & 2.100202 & 0.9058938 & 1.9212934 & 0.0004783741 & $4.603832 e-01$ & $4.816617 e-04$ & 3157 \\
\hline OXCT1 & 1.819297 & 1.9586551 & 3.4500430 & 0.0170407068 & $8.766088 e-03$ & $2.904319 e-04$ & 5019 \\
\hline AACS & 2.469117 & 0.7368114 & 0.9974199 & 0.0001754300 & $2.422253 e-02$ & $9.865016 \mathrm{e}-01$ & 65985 \\
\hline ACAA2 & 4.821347 & 6.2239741 & 7.7910046 & 0.0000104326 & $3.048665 e-06$ & $8.034791 \mathrm{e}-07$ & 10449 \\
\hline ACADS & 1.888099 & 1.6637445 & 2.0115610 & 0.0038712669 & $9.634027 e-03$ & $1.543005 e-03$ & 35 \\
\hline ACADSB & 1.195094 & 2.0069530 & 2.0564667 & 0.2802910307 & $1.433687 e-03$ & $9.039504 e-04$ & 36 \\
\hline ALDH2 & 1.763443 & 3.1268333 & 3.8252809 & 0.0011078739 & $2.567486 e-05$ & $7.090091 \mathrm{e}-06$ & 217 \\
\hline ALDH6A1 & 1.477986 & 2.8764447 & 2.5281127 & 0.2441248953 & $6.354150 e-03$ & $9.714436 \mathrm{e}-03$ & 4329 \\
\hline BCAT2 & 2.316116 & 2.4126245 & 2.5368003 & 0.0001293614 & $6.566339 \mathrm{e}-05$ & $3.177567 e-05$ & 587 \\
\hline BCKDHB & 1.431569 & 1.5486911 & 2.1965202 & 0.0452393293 & $1.806377 \mathrm{e}-02$ & $7.694700 \mathrm{e}-04$ & 594 \\
\hline IVD & 1.549890 & 2.2416537 & 2.8400924 & 0.0080912765 & $3.245635 e-04$ & $5.772394 \mathrm{e}-05$ & 3712 \\
\hline MUT & 1.571621 & 1.5632852 & 2.0246213 & 0.0247733431 & $2.328297 e-02$ & $2.090552 e-03$ & 4594 \\
\hline
\end{tabular}

cysteine and methionine metabolism

\begin{tabular}{|c|c|c|c|c|c|c|c|}
\hline Offical gene symbol & fold ratio $7 D / 24 h$ & fold ratio $15 \mathrm{D} / 24 \mathrm{~h}$ & fold ratio $30 \mathrm{D} / 24 \mathrm{~h}$ & adj.p.value D7/24h & adj.p.value D15/24h & adj.p.value D30/24h & ENTREZ GENE ID \\
\hline CTH & 1.1650536 & 0.6765946 & 0.5735644 & 0.4039507699 & $3.071043 e-02$ & $5.021910 \mathrm{e}-03$ & 1491 \\
\hline LDHA & 1.9544593 & 1.2526597 & 0.7228589 & 0.0004952075 & $5.907971 \mathrm{e}-02$ & $1.094488 \mathrm{e}-02$ & 3939 \\
\hline CDO1 & 0.1697129 & 0.1687964 & 0.1496104 & 0.0006446968 & $4.735026 e-04$ & $2.404164 \mathrm{e}-04$ & 1036 \\
\hline BCAT2 & 2.3161159 & 2.4126245 & 2.5368003 & 0.0001293614 & $6.566339 \mathrm{e}-05$ & $3.177567 e-05$ & 587 \\
\hline GCLC & 0.6880218 & 0.5556420 & 0.4591907 & 0.0358120264 & $4.095234 e-03$ & $7.387924 \mathrm{e}-04$ & 2729 \\
\hline MTR & 0.9431754 & 1.4463115 & 1.9869326 & 0.7488047244 & $2.024648 \mathrm{e}-02$ & $7.361090 \mathrm{e}-04$ & 4548 \\
\hline $\mathrm{AHCY}$ & 0.6856730 & 0.4094371 & 0.1939513 & 0.0313141379 & $4.354019 \mathrm{e}-04$ & $1.257205 \mathrm{e}-05$ & 191 \\
\hline DNMT1 & 0.4334279 & 0.4842405 & 0.2463133 & 0.0003463974 & $5.106746 \mathrm{e}-04$ & $1.147965 \mathrm{e}-05$ & 1786 \\
\hline SRM & 0.4305063 & 0.4535474 & 0.3770184 & 0.0002199534 & $2.066108 e-04$ & $4.638178 e-05$ & 6723 \\
\hline ENOPH1 & 0.6843329 & 0.7252027 & 0.4904430 & 0.0655611883 & $1.046725 e-01$ & $2.981998 \mathrm{e}-03$ & 58478 \\
\hline
\end{tabular}

expressed genes belonging to the "valine, leucine and isoleucine degradation" and "cysteine and methionine metabolism" KEGG pathways. Applied prediction methods that used text mining, co-expression, experimentally observed interactions (Fig. 4).

\section{Discussion}

The oviduct (uterine tube or Fallopian tube) is one of the most specialized organs in the mammalian organism. The oviduct plays a very important role in reproduction, being primarily involved in the transport of oocytes after ovulation. The fertilization process also occurs in the fallopian tube, and thus the oviduct also contributes to the transport of the developing embryo to the uterus [11]-[13]. Under physiological conditions, the fallopian tubes are filled with fluid, which creates the appropriate environment for sperm capacitation, fertilization, early embryonic development [14], [15].

The characteristic feature of oviduct epithelial cells are their cilia, specialised structures that facilitate the transport of the oocyte and / or embryo [16]. The rest of the cells in the OEC are secretory cells, that produce fluid filling the light of the fallopian tube . In in vitro primary culture conditions, OECs display epithelial cell morphology - appearing as densely packed polygonal cells. In the first days of primary culture, ciliated cells are present, disappearing in secondary culture [17]-[19]. Secretory cells are located along the entire length of the fallopian tube in vivo. Their primary function is the production of specific proteins and glycoproteins involved in processes such as gametogenesis, fertilization, early embryonic development. Studies show that there are regional OEC variants, which may reflect distinct functional differences between individual sections of the fallopian tube [12], [20]. On the other hand, many researchers also draw attention to the morphological and functional changes occurring in the fallopian tube epithelium through the menstrual cycle. During follicular growth, in the vesicular phase, OEC cells rapidly proliferate, increase epithelial thickness, and the number of ciliates cells and secretory cells. On the other hand, during the luteal phase, the number of epithelial cells decreases together with OECs secretory activity [21], [22]

Secretory epithelial oviductal cells produce a fluid that contains primarily glycoproteins. Current research confirms that the secreted products have an influence on the correctness of the fertilization process and the early development of the embryo [23], [24]. In recent years many studies have been conducted on in vitro embryonic viability. Many researchers have attempted to use co-cultures, with cells such as human granulosa or various types of stem cells, to achieve the best possible developmental results. There could be a large potential in application of OECs in these kind of studies [25].

Research by Abe et al. was carried out on the bovine model. Studies have shown that bovine OEC, grown in co-cultures with spermatozoa, improve spermatozoa's ability to fertilize oocytes in vitro. It has also been shown that OEC, in in vitro fertilized cows, secrete factors (glycoproteins and growth factors) that have a positive effect on the develop- 
ing embryo. These studies are therefore useful in the analysis of fertility problems and provide many information on how to improve the quality of developing embryos in vitro [22], [26]. Substances secreted by the OECs influence the sperm, improving their ability to fertilise oocytes [20].

The above analyses concern the metabolism of several essential amino acids. These are exogenous amino acids that cannot be synthesized by the animal or human body. The present research concerns cysteine, methionine, valine, leucine and isoleucine [27].

Cysteine and methionine belong to the group of amino-acids consisting sulphur. Methionine is an essential amino acid that is not synthesized by the animal and human organism. In the case of plants and bacteria, methionine is synthesized from aspartate. Methionine, by combining with ATP, is involved in the formation of S-Adenosylmethionine (SAM). Furthermore, SAM is a donor of methyl groups during DNA methylation, and thus participates in regulation of gene expression [28], [29].

Our study indicates that during long-term, in vitro breeding of pig oviduct epithelial cells, major changes in genes, regulating expression of leucine and isoleucine degradation, occur. The vast majority of these genes exhibit increased expression on day 30 of the culture. So far, no change in the expression of these individual genes has been studied in oviductal cells.

The branched-chain amino acids (BCAA) is a group of amino acids that exhibit chain branching. There are 3 members of this group: leucine, isoleucine and valine. Group of this amino acids cannot be synthetized in human and other mammals, they must be provided through dietary means. BCAA is a group of compounds that are extensively studied in human and animal models. Most of the research conducted focuses on the influence of these amino acids on physical fitness, primarily of men [30]. They are mainly associated with mediation of weight loss [31]. ACAA2 (Acetyl-CoA Acyltransferase 2 or mitochondrial acetyl-CoA acyltransferase) is one of the five types of thiolases found in mammals. ACAA2 is responsible for catalysis of the last step of the mitochondrial fatty acid beta-oxidation spiral [32].

Branched-chain keto acid dehydrogenase complex (BCKD) is involved in the second stage of BCAA catabolism. This complex consists of three catalytic components: a heterotetrameric (alpha2, beta2) branched-chain alpha-keto acid decarboxylase (E1), homo-24-dihydrolipoly transacylase (E2), and a homodimeric dihydrolipoamide dehydrogenase (E3). The second stage of the BCAA catabolism is an irreversible stage in the BCAA oxidation process. BCKS has two regulatory enzymes (kinase and phosphorylase) [33], [34].

Another gene involved in the pathway of degradation of valine, leucine and isoleucine, whose expression has clearly increased over the 30-day in vitro culture, is 3-oxoacid-CoA transferase (OXCT1).
This gene is responsible for the proper synthesis of succinyl-CoA or 3-ketoacid CoA transferase (SCOT). Ketones such as acetylacetate, 3-hydroxybutyrate and acetone, primarily produced by the liver, are the primary source of energy for the brain when the glucose content in the body decreases. Any disturbance of the ketolysis process can lead to excessive accumulation of ketones in the body and thus cause ketoacidosis [35], [36]. SCOT is produced in the mitochondria, primarily in the liver, liver during fat breakdown. Ketone bodies are an important source of energy during difficult conditions and at the times of increasing energy requirements such as: fasting, diet and intense physical activity. SCOT is involved in acetoacetate to acetoacetyl-CoA catalysis [37]. Acetoacetyl-CoA is metabolized by the thiolase of acetoacetyl-CoA to two acetyl-CoA molecules. These two molecules are the substrate in the citric acid cycle, when producing energy [38]. Tanak et al. have recognised the expression of 3-oxo-acid CoA transferase (scot-t), as characteristic for reproductive cells. The gene responsible for the synthesis of this enzyme was expressed in sperm mitochondria [39]. Therefore it is safe to assume that the expression of the OXCT1 gene occurs not only in the testes, but also in the cells of the oviduct. Another gene that exhibits increased expression during the 30 days of primary culture is the gene responsible for isovaleryl-CoA dehydroganase (IVD) sythesis. IVD is an enzyme belonging to the acyl-CoA dehydrogenase I group and participates in the leucine catabolism [40], [41]. Similar expression levels are shown by 2 genes representing the aldehyde dehydrogenase family. The first of these two genes is acetaminophen dehydrogenase 2 (ALDH2). This gene encodes one of the most important enzymes involved in the metabolism of alcohol . In addition, Chen et al. They found a mitochondrial ALDH2, whose activity was correlated with decreased ischemic heart damage in rodents. ALDH2 is most likely to reduce the production of cytotoxic aldehydes during infarction [42].

The second gene is aldehyde dehydrogenase 6 family member A1 gene (ALDH6A1). This gene is responsible for the synthesis of methylmalonate semialdehyde dehydrogenase, enzyme that catalyzes the oxidative decarboxylation of malonate and methylmalonate semialdehydes to acetyl- and propionyl-CoA, respectively [43], [44].

The gene, that is also involved in the BCAA degradation pan, is the AACS gene encoding the cytosolic ligase. This enzyme participates in ketone body acetoacetate activation to form CoAs during cholesterol and fatty acid synthesis. The role of AACS in mammals is not fully known. Expression of the gene responsible for AACS synthesis was found to be high in human brain, kidney, and heart and low in liver [45]. Our results indicate that AACS expression was low on the first day of culture, then increased during day 7 of culture, followed by a decrease on day 30 . 
Another pathway described in this work is cysteine and methionine metabolism. Studies have shown that activation of this pathway occurs in the long-term in vitro OEC culture. Expression of most of the presented genes showed a decline on day 30 of the culture. Genes such as spermidine (SRM), CD01, ENOPH show the highest decrease in expression during the first 7 days of culture. Interestingly, genes such as AHCY, GCLS, CTH show decreased expression only on day 15 of the culture.

Rapid decrease in expression of SRM was observed. SRM belongs to the family of polyamines, playing an important role in the growth, proliferation and cell survival. Many authors point out that the level of spermidine in cells falls with age. These studies also suggest that in vitro retarded cells exhibit a decrease in SRM expression [46]. Eisenberg et al. showed that spermidine administration inhibited yeast, fly and human cell longevity, by increasing autophagy, but this is also related to reduced oxidative stress [47]. Another gene showing downregulation after 7 days of culture is enolase-phosphatase 1 (ENOPH1). This enzyme is released during methionine metabolism and regulates the stress response of cells. Overexpression or complete blockage of ENOPH1 resulted in increased cell mortality [48].

These results suggest that during long-term in vitro OEC culture the pathways of amino acid metabolism characteristic for other cell types are activated. These studies are among the first to show the genes involved in the metabolism of individual amino acids, which may therefore be genetic markers of the processes involved in amino acid metabolism in OEC-specific long-term in vitro culture.

Referring to our findings on the results, obtained by Sen et al., expression DNMT1 has a strong influence on the function of the stem cell. Sen et al. have suggested that the gene presented encodes proteins characteristic for undifferentiated cells. The effects of DNMT1 expression decreased with cell differentiation [49]. The close association of DNMT1 with OCT4 and Nanog genes has also been demonstrated, and thus the decrease in expression of these two genes results in a decrease in DNMT1 expression during differentiation. It has been found that this gene plays a fundamental role in regulating stem cell differentiation, primarily that of pluripotent stem cells [50]-[53]. Our studies have shown that DMNT1 expression in long-term in vitro culture decreases. The appearance of DMNT1 expression in the OEC may indicate that these cells possess stem cell potential that decreases with each day of in vitro culture.

\section{Conclusions}

These studies confirm the key role of oviductal epithelial cells in reproduction, while also highlighting the additional properties of OECs. Having the characteristics of stem cells, and thus using this property to obtain diverse, stable cultures of differentiated cells that could be used in widely understood regenerative medicine.

\section{Acknowledgements}

Publication of this article was made possible by grant number UMO-2016/21/B/NZ9/03535 from Polish National Centre of Science and 502-14-02227367-10694 from Poznan University of Medical Sciences

\section{Author details}

Bartosz Kempisty PhD, Department of Histology and Embryology, Department of Anatomy, Poznań University of Medical Sciences, 6 Święcickiego St., 60-781 Poznań, Poland Tel./Fax: +48 618546418 /+48 61 8546440, e-mail: bkempisty@ump.edu.pl

\section{Conflicts of Interest}

The authors declare they have no conflict of interest

\section{References}

1. Kranc, W. et al., "The origin, in vitro differentiation, and stemness specificity of progenitor cells.," J. Biol. Regul. Homeost. Agents, vol. 31, no. 2 , pp. 365-369, 2017.

2. Groth, C. G., "The potential advantages of transplanting organs from pig to man: A transplant Surgeon's view.," Indian J. Urol., vol. 23, no. 3, pp. 305-9, Jul. 2007.

3. Denner, J., "Xenotransplantation-Progress and Problems: A Review," J. Transplant. Technol. Res., vol. 4, no. 2, Jul. 2014.

4. Kranc, W. et al., "Molecular basis of growth, proliferation, and differentiation of mammalian follicular granulosa cells.," J. Biol. Regul. Homeost. Agents, vol. 31, no. 1, pp. 1-8, 2017.

5. Kempisty, B. et al., "Association between progesterone and estradiol-17beta treatment and protein expression of pgr and PGRMC1 in porcine luminal epithelial cells: a real-time cell proliferation approach.," J. Biol. Regul. Homeost. Agents, vol. 29, no. 1, pp. 39-50.

6. Bukowska, D. et al., "Differential expression of epidermal growth factor and transforming growth factor beta isoforms in dog endometrium during different periods of the estrus cycle," Pol. J. Vet. Sci., vol. 14, no. 2 , pp. 259-264, Jan. 2011.

7. Ciesiółka, S. et al., "Epithelialization and stromalization of porcine follicular granulosa cells during real-time proliferation - a primary cell culture approach.," J. Biol. Regul. Homeost. Agents, vol. 30, no. 3, pp. 693-702.

8. Huang, D. W. et al., "DAVID Bioinformatics Resources: expanded annotation database and novel algorithms to better extract biology from large gene lists.," Nucleic Acids Res., vol. 35, no. Web Server issue, pp. W16975, Jul. 2007.

9. Luo, W. and Brouwer, C., "Pathview: an R/Bioconductor package for pathway-based data integration and visualization," Bioinformatics, vol. 29, no. 14, pp. 1830-1831, Jul. 2013.

10. von Mering, C. et al., "STRING: known and predicted protein-protein associations, integrated and transferred across organisms," Nucleic Acids Res., vol. 33, no. Database issue, pp. D433-D437, Dec. 2005.

11. Pectasides, D., Pectasides, E., and Economopoulos, T., "Fallopian tube carcinoma: a review.," Oncologist, vol. 11, no. 8, pp. 902-12, Sep. 2006.

12. Chen, S., Einspanier, R., and Schoen, J., "Long-term culture of primary porcine oviduct epithelial cells: Validation of a comprehensive invitro model for reproductive science," Theriogenology, 2013.

13. Hunter, R. H. F., "Oviduct function in pigs, with particular reference to the pathological condition of polyspermy," Mol. Reprod. Dev., vol. 29, no. 4, pp. 385-391, Aug. 1991.

14. Jansen, R. P. S., Anderson, J. C., and Sutherland, P. D., "Nonoperative Embryo Transfer to the Fallopian Tube," N. Engl. J. Med., vol. 319, no. 5, pp. 288-291, Aug. 1988.

15. Murray, S. C. and Smith, T. T., "Sperm interaction with fallopian tube apical membrane enhances sperm motility and delays capacitation," Fertil. Steril., vol. 68, no. 2, pp. 351-357, Aug. 1997.

16. Joshi, M. S., "Isolation, cell culture, and characterization of oviduct epithelial cells of the cow," Microsc. Res. Tech., vol. 31, no. 6, pp. 507-518, Aug. 1995.

17. Joshi, M. S., "Isolation, cell culture and immunocytochemical characterization of oviduct epithelial cells of the cow.," J. Reprod. Fertil., vol. 83, no. 1, pp. 249-61, May 1988.

18. Walter, I., "Culture of bovine oviduct epithelial cells (BOEC)," Anat. Rec., vol. 243, no. 3, pp. 347-356, Nov. 1995. 
19. Aldarmahi, A., "Establishment and characterization of female reproductive tract epithelial cell culture," J. Microsc. Ultrastruct., vol. 5, no. 2, pp 105-110, Jun. 2017.

20. Rottmayer, R. et al., "A bovine oviduct epithelial cell suspension culture system suitable for studying embryo-maternal interactions: morphological and functional characterization.," Reproduction, vol. 132, no. 4, pp. 637-48, Oct. 2006

21. Leese, H. J., Tay, J. I., Reischl, J., and Downing, S. J., "Formation of Fallopian tubal fluid: role of a neglected epithelium.," Reproduction, vol. 121, no. 3 , pp. 339-46, Mar. 2001.

22. Abe, H. and Oikawa, T., "Observations by scanning electron microscopy of oviductal epithelial cells from cows at follicular and luteal phases," Anat. Rec., vol. 235, no. 3, pp. 399-410, Mar. 1993.

23. Kress, A. and Morson, G., "Changes in the oviducal epithelium during the estrous cycle in the marsupial Monodelphis domestica., J. Anat., vol. 211, no. 4, pp. 503-17, Oct. 2007

24. Huntar, R. H., "Function and malfunction of the Fallopian tubes in relation to gametes, embryos and hormones.," Eur. J. Obstet. Gynecol. Reprod. Biol., vol. 7, no. 4, pp. 267-83, 1977.

25. Kossowska-Tomaszczuk, K. et al., "The Multipotency of Luteinizing Granulosa Cells Collected from Mature Ovarian Follicles," Stem Cells, vol. 27, no. 1, pp. 210-219, Jan. 2009.

26. Abe, H. and Hoshi, H., "Bovine oviductal epithelial cells: their cell culture and applications in studies for reproductive biology.," Cytotechnology, vol. 23, no. 1-3, pp. 171-83, Jan. 1997.

27. McGivan, J. D. and Pastor-Anglada, M., "Regulatory and molecular aspects of mammalian amino acid transport.," Biochem. J., vol. 299 ( Pt 2), no. Pt 2, pp. 321-34, Apr. 1994

28. Finkelstein, J. D., Martin, J. J., and Harris, B. J., "Methionine metabolism in mammals. The methionine-sparing effect of cystine.," J. Biol. Chem., vol. 263, no. 24, pp. 11750-4, Aug. 1988

29. Stadtman, E. R., Van Remmen, H., Richardson, A., Wehr, N. B., and Levine, R. L., "Methionine oxidation and aging," Biochim. Biophys. Acta - Proteins Proteomics, vol. 1703, no. 2, pp. 135-140, Jan. 2005.

30. Zhang, S., Zeng, X., Ren, M., Mao, X., and Qiao, S., "Novel metabolic and physiological functions of branched chain amino acids: a review.," $J$ Anim. Sci. Biotechnol., vol. 8, p. 10, 2017.

31. Wolfe, R. R., "Branched-chain amino acids and muscle protein synthesis in humans: myth or reality?," J. Int. Soc. Sports Nutr., vol. 14, p. 30, 2017.

32. Abe, H. et al., "Cloning and sequence analysis of a full length cDNA encoding human mitochodrial 3-oxoacyl-CoA thiolase," Biochim. Biophys. Acta-Gene Struct. Expr., vol. 1216, no. 2, pp. 304-306, Nov. 1993.

33. Harris, R. A. et al., "Regulation of the branched-chain alpha-ketoacid dehydrogenase and elucidation of a molecular basis for maple syrup urine disease.," Adv. Enzyme Regul., vol. 30, pp. 245-63, 1990.

34. Wynn, R. M. et al., "Molecular Mechanism for Regulation of the Human Mitochondrial Branched-Chain $\alpha$-Ketoacid Dehydrogenase Complex by Phosphorylation," Structure, vol. 12, no. 12, pp. 2185-2196, Dec. 2004.

35. Shafqat, N. et al., "A structural mapping of mutations causing succinyl-CoA:3-ketoacid CoA transferase (SCOT) deficiency," J. Inherit. Metab. Dis., vol. 36, no. 6, pp. 983-987, Nov. 2013

36. Cotter, D. G., Schugar, R. C., and Crawford, P. A., "Ketone body metabolism and cardiovascular disease.," Am. J. Physiol. Heart Circ. Physiol., vol. 304 no. 8, pp. H1060-76, Apr. 2013.

37. Fukao, T. et al., "Succinyl-CoA:3-Ketoacid CoA Transferase (SCOT): Cloning of the Human SCOT Gene, Tertiary Structural Modeling of the Human SCOT Monomer, and Characterization of Three Pathogenic Mutations," Genomics, vol. 68, no. 2, pp. 144-151, Sep. 2000.

38. Williamson, D. H., Bates, M. W., Page, M. A., and Krebs, H. A., "Activities of enzymes involved in acetoacetate utilization in adult mammalian tissues.," Biochem. J., vol. 121, no. 1, pp. 41-7, Jan. 1971.

39. Tanaka, H., Kohroki, J., Iguchi, N., Onishi, M., and Nishimune, Y., "Cloning and characterization of a human orthologue of testis-specific succinyl CoA: 3-oxo acid CoA transferase (Scot-t) cDNA," Mol. Hum. Reprod., vol. 8, no. 1, pp. 16-23, Jan. 2002.

40. Matsubara, Y. et al., "Molecular cloning and nucleotide sequence of cDNAs encoding the precursors of rat long chain acyl-coenzyme A, short chain acyl-coenzyme A, and isovaleryl-coenzyme A dehydrogenases. Sequence homology of four enzymes of the acyl-CoA dehydrogenase family.," J. Biol. Chem., vol. 264, no. 27, pp. 16321-31, Sep. 1989.

41. Bixel, M. G., Shimomura, Y., Hutson, S. M., and Hamprecht, B., "Distribution of Key Enzymes of Branched-chain Amino Acid Metabolism in Glial and Neuronal Cells in Culture," vol. 49, no. 3, pp. 407-418, 2001.

42. Chen, C.-H., Budas, G. R., Churchill, E. N., Disatnik, M.-H., Hurley, T. D., and Mochly-Rosen, D., "Activation of Aldehyde Dehydrogenase-2 Reduces Ischemic Damage to the Heart," Science (80-. )., vol. 321, no. 5895, pp. 1493-1495, Sep. 2008
43. Kedishvili, N. Y., Popov, K. M., Rougraff, P. M., Zhao, Y., Crabb, D. W., and Harris, R. A., "CoA-dependent methylmalonate-semialdehyde dehydrogenase, a unique member of the aldehyde dehydrogenase superfamily: cDNA cloning, evolutionary relationships, and tissue distribution," $J$. Biol. Chem., vol. 267, no. 27, pp. 19724-19729, 1992.

44. Allahverdiyev, M., A. et al., "Aldehyde Dehydrogenase: Cancer and Stem Cells," in Dehydrogenases, InTech, 2012.

45. Ohgami, M., Takahashi, N., Yamasaki, M., and Fukui, T., "Expression of acetoacetyl-CoA synthetase, a novel cytosolic ketone body-utilizing enzyme, in human brain.," Biochem. Pharmacol., vol. 65, no. 6, pp. 989-94, Mar. 2003.

46. Minois, N., "Molecular basis of the 'anti-aging' effect of spermidine and other natural polyamines - a mini-review.," Gerontology, vol. 60, no. 4, pp. 319-26, 2014

47. Eisenberg, T. et al., "Induction of autophagy by spermidine promotes longevity," Nat. Cell Biol., vol. 11, no. 11, pp. 1305-1314, Nov. 2009.

48. Zhang, Y. et al., "Cerebral Microvascular Endothelial Cell Apoptosis after Ischemia: Role of Enolase-Phosphatase 1 Activation and Aci-Reductone Dioxygenase 1 Translocation," Front. Mol. Neurosci., vol. 9, p. 79, Aug. 2016.

49. Sen, G. L., Reuter, J. A., Webster, D. E., Zhu, L., and Khavari, P. A., "DNMT1 maintains progenitor function in self-renewing somatic tissue," Nature, vol. 463, no. 7280, pp. 563-567, Jan. 2010.

50. PAN, G. J., CHANG, Z. Y., SCHÖLER, H. R., and PEI, D., "Stem cell pluripotency and transcription factor Oct4," Cell Res., vol. 12, no. 5-6, pp. 321 329, Dec. 2002

51. Shi, G. and Jin, Y., "Role of Oct4 in maintaining and regaining stem cell pluripotency," Stem Cell Res. Ther., vol. 1, no. 5, p. 39, Dec. 2010.

52. Pan, G. and Thomson, J. A., "Nanog and transcriptional networks in embryonic stem cell pluripotency," Cell Res., vol. 17, no. 1, pp. 42-49, Jan 2007.

53. Schwarz, B. A., Bar-Nur, O., Silva, J. C. R., and Hochedlinger, K., "Nanog Is Dispensable for the Generation of Induced Pluripotent Stem Cells," Curr Biol., vol. 24, no. 3, pp. 347-350, Feb. 2014. 